Japanese Psychological Research

1986, Vol. $28, \mathrm{No} .2,94-103$

\title{
Conservative cutoff placement in a numerical decision task $^{\mathrm{i}}$
}

\author{
YUJI HOSHINO \\ Department of Psychology, Faculty of Social Sciences and Humanilies, Tokyo Metropolitan University, \\ Meguro-ku, Tokyo 152
}

\begin{abstract}
Conservative placement of a criterion was investigated in a numerical decision task where the subjects were required to make binary decisions on five-digit numbers drawn from two overlapping normal distributions. In Experiment 1 (subjects were 32 graduates and undergraduates), there were two conditions of discriminability determined by the degree of the overlap of distributions. Although observed cutoff locations for uneven prior probabilities of stimulus distributions were conservative in both conditions, the subjects in the low discriminative condition changed their cutoff placement less than those in the high discriminative condition. In both conditions, subjects' response probabilities were close to the prior probabilities. In Experiment 2 (subjects were eight graduates and undergraduates), the verbal protocol during the task showed that ambiguous impressions were used for judgments and that responses were made as accurately as possible to both stimulus distributions. It was concluded that the heuristics the subjects used in the task could account for the conservative cutoff placement and that the heuristics confirmed their judgments.
\end{abstract}

Key words: heuristics, decision making, categorization, signal detection theory, probability matching, conservatism (decision making), criteria.

Signal detection theory could be regarded as a model of decision containing uncertainty. In this theory, a likelihood ratio of two overlapped normal distributions, which is called $\beta$, indicates a location of a criterion at which the decision is assumed to change. The optimal value of $\beta$ which maximizes reward and minimizes cost is determined by prior probabilities of stimulus sets and a payoff matrix.

Conservative cutoff placement of observed $\beta$ has been found in a signal detection task (Green \& Swets, 1966), as well as in a discrimination task (Ulehla, 1966), a recognition memory task (Healy \& Kubovy, 1977, 1978), and a numerical decision task (Healy \& Kubovy, 1981; Hoshino, 1982). Although observed $\beta$ exhibits the appropriate response bias for

\footnotetext{
1 Part of this research was presented at the 49 th Annual Canvention of the Japanese Psychological Association, Tokyo, July, 1985.

a I thank K. Morigiwa for her careful reading of this manuscript.
}

uneven prior probabilities and/or an asymmetrical payoff matrix, the absolute value of the shift in the observed $\beta$ is smaller than that required for the shift to the optimal $\beta$. Healy and Kubovy (1981) changed the value of the optimal $\beta$ from $1 / 9$ to 9 , and found the conservative cutoff placement. In addition, they reported a response strategy called probability matching. Subjects have a tendency to match their response probabilities to the prior probabilities of stimulus categories (Parks, 1966).

In this study, the numerical decision task was used to investigate the cause of the conservative cutoff placement. In the task, which is considered to be a probabilistic categorization task, subjects made a response to a presented digital stimulus derived from two overlapped normal distributions, and the response was chosen from two arbitrary categories corresponding to the stimulus distributions. After each trial, feedback as to whether subjects' response was correct or not was given to 
them. By using numbers as stimuli, the physical effect of stimulus presentation and the physiological effect involved in the sensory process can be ignored (Kubovy \& Healy, 1977b).

The relation between $d^{\prime}$, a discriminability of overlapped normal distributions, and $\ln , \hat{\gamma}$ (natural logarithm of $\beta$ ) is as follows when $z$-scores of false alarm rates are denoted by $z$ :

$$
\ln \beta=\frac{1}{2} d^{\prime}\left(2 z-d^{\prime}\right) .
$$

Let us define $k$ as representing the difference between the cutoff point and the midpoint of the two means of the distributions,

$$
k=z-\frac{1}{2} d^{\prime}
$$

Then, Equation 1 can be rewritten:

$$
\ln \beta=k d^{\prime} .
$$

The larger the value of $d^{\prime}$, the larger the change in $\ln \beta$. Winterfeldt and Edwards (1982) show that when prior probabilities of stimulus distributions are even, the steepest functions of the expected values of payoff subjects could gain occur between $d$ 's of 2 and 3. At the same time, they point out that " for all levels of $d$ ' a difference of one standard deviation between the optimal and the actual decision criterion would result in a loss of only about $20 \%$ or less" (p. 618).

Violations of a fixed cutoff point have been observed in other numerical decision tasks (Kubovy \& Healy, 1977a; Kubovy, Rapoport, \& Tversky, 1971; Ward, 1973). These were responses which contradicted the hypothesized static criterion. Ward (1973) found that these violations were concentrated in the region where the subjects were likely to place their criterion and that the subjects could use the firstorder stimulus dependencies for their judgments. These results suggest that information about the preceding stimuli affect decision making, especially when the decision is uncertain. A threc-state two-threshold model (Sandusky, 1971), a criterion band model (Ward, 1973), and a dynamic cutoff model (Kubovy \& Healy, 1977a; Treisman \& Williams, 1984) were proposed for the sequential dependencies and the cutoff violations. If stimuli are presented randomly, unsystematic attempts to use sequential information would lower the value of observed $d^{\prime}$. Such behavior should often show up as violations of a fixed cutoff point (Ward, 1973).

In this study, asymmetrical prior probabilities of stimulus distributions were used to shift the location of the optimal $\beta$. In Experiment 1, there were two conditions of discriminability determined by the degree of overlap of two stimulus distributions. It would be easier for the subjects in the high discriminative condition to shift their criterion $(\beta)$ than those in the low discriminative condition. In addition, several indices of subjects' performance were measured, revealing quantitative properties of the task. Based on the results of Experiment 1, Experiment 2 was to examine subjects' heuristics. Verbal protocols of the task were used for detailed analyses of the conservative cutoff placement. Since the subjects should have strategies for uncertain judgments in the task, their strategies would be manifested in their protocols.

\section{Experiment 1}

\section{Method}

Subjects and apparatus. Thirty-four graduates and undergraduates, who had never experienced the numerical decision task, participated in this experiment. They were randomly allocated to the low or the high discriminative condition. Two of the subjects assigned to the low discriminative condition were excluded because their performance were at the level of chance during the first half of the sessions. Data collected from the remain- 
ing 32 subjects were reported, and each of the two conditions contained eight male and eight female subjects. A Fujitsu FM-8 personal computer with a green CRT display was used to present the stimuli.

Stimuli. Four sets of 84 five-digit numbers forming appropriate normal distribution were constructed, and there was no identical number in the set. Each of the four sets was divided into a pair of subsets. In two of the four pairs, any subset contained 42 five-digit numbers. In the other two pairs, one subset of the pair contained 21 five-digit numbers, and the other subset contained 63. All of the subsets had approximate normal distributions with a mean of 54000 and a standard deviation of 3000 . To make the normal distributions overlap, a constant value was subtracted from each of the numbers in one subset of the pair, and the same constant value was added to the numbers in the other subset. The constant value was 1200 for the low discriminative condition, and 2700 for the high discriminative condition, the value of $d^{\prime}$ of the overlapped normal distributions for the low discriminative condition being .8 , and that for the high discriminative condition being 1.8 .

Design and procedure. Both low and high discriminative conditions contained four sessions, consisting of 84 trials each. The two symmetrical pairs of subsets of fivedigit numbers were used in the first two sessions, and the two asymmetrical pairs were used in the latter two.

To become familiar with the apparatus, the subjects were given 20 practice trials responding to the direction of a horizaontal array on the CRT. In the subsequent numerical decision task, the five digit numbers were used to represent the heights of men or women. 'The correspondence between the normal distribution and the heights of humans was illustrated to the subjects, displaying the density functions of two overlapped normal distributions on the CRT before instructions.

The instructions about the numerical decision task were as follows: "Fortytwo males and 42 females will appear. If you push the space bar key, the instruction on the CRT will disappear, and after one second a five-digit number will appear. Let the number represent the height of human beings measured by an arbitrary and unreal unit, and if you think it represents a man's height, push the '.' key with your middle finger, if you think it represents a woman's, push the ' 0 ' key with your index finger. When you have made your decision, please respond as immediately and correctly as possible. The correctness or the incorrectness of your response will subsequently appear on the CRT, please take a look at it and proceed to the next trial by pushing the space bar key again. You can keep your own pace." During the interval of Sessions 2 and 3 , the subjects were told that the third session would be in the same condition as in the second session except that the heights of 21 males and 63 females were to appear. Both total numbers of male-heights and female-heights presented in each session were displayed on the CRT throughout the trials so that the subjects would not forget the numbers. In the other two intervals between Sessions 1 and 2 , and 3 and 4 , the subjects were told that the second or fourth session would be in the same condition as in the last one, and to perform in the same way as they had in the last session.

When one of the response keys was pushed, the presented stimulus disappeared and a brief buzzer tone came on. When the response made by the subjects was correct, they gained one point. In each session, the sum of the points subjects got up to that trial was displayed on the CRT after each trial. Minimum time between displays of a stimulus was set to three seconds. The presentation order of the stimuli was randomized for each subject. Four combinations of the four pairs of the 
Table 1

Mean vaules of observed $\beta$, critical point, and $\rho$ (Man)

\begin{tabular}{|c|c|c|c|c|c|}
\hline & \multirow{2}{*}{ Condition ${ }^{n}$} & \multicolumn{4}{|c|}{ Session } \\
\hline & & 1 & 2 & 3 & 4 \\
\hline \multirow[t]{2}{*}{$\beta$} & Low & 1.03 & 1.05 & 1.45 & 1.40 \\
\hline & High & .90 & .89 & 1.95 & 2.01 \\
\hline \multirow[t]{2}{*}{ Critical point } & Low & 54354 & $5+263$ & 55882 & 55803 \\
\hline & Higlı & 53510 & 53825 & 55615 & 55427 \\
\hline \multirow[t]{2}{*}{$p$ (Man) } & Low & .48 & .47 & .24 & .25 \\
\hline & High & .54 & .51 & .24 & .24 \\
\hline
\end{tabular}

8) Two conditions between subjects are low $\left(d^{\prime}=1.8\right.$ for the ideal observer) and high discriminability $\left(d^{\prime}=.8\right.$ for the ideal observer).

subsets were made to counterbalance the order effect of the stimulus sets. It took about half an hour to complete this experiment.

\section{Results and Discussion}

Statistical significance was judged by .05 of the one-tailed probability in the following analyses. Analyses of variance on observed $d^{\prime}$ revealed that only the effect of condition was significant, $F(1,30)$ $=206.6, p<.01$. The average values of observed $d^{\prime}$ for the low and high discriminative conditions were .69 and 1.51 , respectively. These conditions are referred to as the low condition and the high condition in the following. The means of the total points subjects gained over the four sesseions were 226.7 in the low condition, and 266.0 in the high condition. The expected value of the total points the subjects could gain was 246.0 in the low condition, and 288.0 in the high condition. These expected values were calculated results for each stimulus distribution presented. The range of differences between the means of the points subjects gained and the expected values was between 2.7 and 7.4 per session.

The values of observed $\beta$ were transformed by a natural logarithm for subsequent analyses. The average values of observed $\beta$ were shown in Table 1 . In Sessions 3 and 4 where asymmetrical prior probabilities were used, the mean values of observed $\beta$ were significantly smaller than 3.0 , which was the value of the optimal $\beta ; t(15)=21.8$ and $t(15)=12.8$, respectively, in the low condition, and $t(15)=6.0$ and $t(15)=4.8$, respectively, in the high condition; for all $t$-values, $p<.01$. The interaction of session and condition was significant $[F(3,90)=13.1, p<.01]$, in addition to the effect of condition $[F(1,30)$ $=68.8, p<.01]$. Separate analyses of variance revealed that there was no significant effect in Sessions 1 and 2, and that in Sessions 2 and 3 the effect of session and the interaction of session and condition were significant $[F(1,30)=129.7, p<.01$, and $F(1,30)=23.0, p<.01$, respectively], and that in Sessions 3 and 4 only the effect of condition was significant $[F(1,30)$ $=19.6, p<.01]$. Changes in the observed $\beta$ for the asymmetrical prior probabilities were larger in the high condition than in the low condition.

A different index of subjects' cutoff location was proposed by Kubovy and Healy (1977a, pp. 432-433). The calculation procedure of this index, called a critical point, is as follows. Five-digit numbers presented in a session are sorted in order of their magnitude. Provided that the midpoint of two successive fivedigit numbers is a criterion, the number of responses which are in contradiction to the criterion is added up, and this 
calculation was made across all of the midpoints. The minimum of these numbers is called the number of static-cutoff violations, and the midpoint for which the number of the static-cutoff violations is calculated is the critical point. Table 1 shows the critical points averaged over subjects for each session. The means of the optimal critical points that were calculated for the stimulus distributions presented in Sessions 3 and 4 was 57922 for the low condition, and 55875 for the high condition. The effects of condition and session were significant, $F(1,30)=$ $5.6, p<.05$ and $F(3,90)=76.9, p<.01$, respectively. The interaction of condition and session was not significant. These results show that although the subjects in the low condition needed to make a larger shift of their cutoff locations, they changed their cutoff locations no more than those in the high condition.

The average numbers of the staticcutoff violations for the first, second, third, and fourth sessions were 9.7, 7.3, 5.4, and 6.4 , respectively, in the low condition, and $10.3,6.8,5.3$, and 4.3 , respectively, in the high condition. The effect of session was significant, $F(3,90)=11.5, p<.01$. In the latter two sessions, the numbers of the static-cutoff violations were fewer than those in the first two.

If the subjects were to have a standard five-digit number for their judgments, reaction times to borderline stimuli would be expected to be longer, because of the uncertainty of that standard number. This increment of the reaction times might also result from the distance effect, that is, the smaller the difference between two numbers, the longer the time required for judgment (Sekuler, Rubin, \& Armstrong, 1971). Five-digit numbers that were presented in the second session and reaction times of correct responses in that same session were used in the following analyses. The five-digit numbers belonging to the same distribution were categorized into the larger and the smaller numbers. The numbers of each distribution were categorized by the point displacing the mean of the distribution by one eighth probability away from the midpoint of the two distributions. This procedure makes the numbers of correct responses to the small and to the large stimuli close to each other in both conditions. The mean reaction times to the large and the small stimuli of male and female heights for each subject were averaged. For the female-height stimuli, the averaged reaction times to the large-number stimuli were significantly longer than those to the small-number stimuli, $F(1,15)=11.1$, $p<.01$ in the low condition and $F(1,15)=$ $11.0, p<.01$ in the high condition. For the male-height stimuli, the averaged reaction times to the small-number stimuli were significantly longer than those to the large-number stimuli, $F(1,15)=13.2, p<$ .01 in the low condition and $F(1,15)=$ $18.1, p<.01$ in the high condition. These differences were about $200 \mathrm{~ms}$. Reaction times to the borderline stimuli were longer, even in the second session under the same condition as the first except for the stimulus sets.

Probabilities of making a response of a man's height regardless of its correctness or incorrectness, to be called $p$ (Man), were averaged over the subjects and the mean values of observed $p(\operatorname{Man})$ are shown in Table 1. The effect of session was significant, $F(3,30)=312.3, \quad p<.01$ and the interaction of condition and session was also significant, $F(3,90)=3.0, p<.05$. In Sessions $l$ and 2, only the effect of condition was significant, $F(1,30)=5.6$, $p<.05 .^{3}$ In Sessions 2 and 3 , only the effect of session was significant, $F(1,30)=$ $385.1, p<.01$. In Sessions 3 and 4 , there was no significant effect. In the latter

3 This significant difference might be caused by the stimulus effect. The mean of the optimal critical points calculated for the stimulus distributions presented in Sessions 1 and 2, was 54643 for the low discriminative condition and 53517 for the high discriminative condition. 
two sessions of both conditions, the mean values of observed $p(\mathrm{Man})$ were close to the prior probability of male-height stimuli. The means of the optimal values of $p$ (Man), calculated for the stimulus distributions presented in the latter two sessions of the low and the high condition, were .08 and .20 , respectively. The differences between the mean values of observed $p$ (Man) and the optimal values in Sessions 3 and 4 were significant; $t(15)=10.3, p<.01$ and $t(15)=8.2, p<.01$, respectively, in the low condition, and $t(15)=3.9, p<.01$ and $t(15)=3.1, p<.01$, respectively, in the high condition.

Kubovy and Healy (1977a) made the subjects report a five-digit number as a criterion before a number to be judged was displayed. In Experiment 2 of the present study, thinking-aloud method was used. Verbal protocols have possibility of unveiling subjects' internally heeded cues and internal responses (Ericsson \& Simon, 1980). Does the conservative cutoff placement reflect nothing but the idleness of the subjects? The aim of Experiment 2 was to examine the heuristics the subjects used to replace their criterion for asymmetrical prior probabilities.

\section{Experiment 2}

\section{Method}

Subjects, stimuli, and apparatus. Eight graduates and undergraduates (four males and four females) participated, experiencing the numerical decision task for the first time in this experiment. The four pairs of subsets of five-digit numbers used in Experiment 1 were utilized. One thousand five hundred was added to each five-digit number in one subset of the pair to represent a male-height distribution, and from each five-digit number in the other subset 1500 was subtracted to represent a female-height distribution, the value of $d^{\prime}$ of the stimulus distributions being 1.0. The apparatus were the same as those in Experiment 1.
Table 2

Mean values of observed $d^{\prime}, \beta$, and $p$ (Man)

\begin{tabular}{cccc}
\hline Sessions & $d^{\prime}$ & $\beta$ & $p$ (Man) \\
\hline 1 & .84 & 1.08 & .48 \\
2 & .74 & 1.57 & .23
\end{tabular}

Design and procedure. Two sessions of 84 trials were conducted, and the subjects were required to verbalize in Japanese throughout the sessions. In the first session, prior probabilities of stimuli were even, and in the second session, asymmetrical prior probabilities were used. Twenty-one male and 63 female heights appeared in the second session.

The instructions were the same as those in Experiment 1 except that the subjects were required to verbalize anything they thought or felt during the numerical dicision task, instead of demanding them an immediate response when they had made their decision. The subjects were prompted to verbalize when they spoke very little during the trials of the task. The subjects' utterance and the buzzer tone triggered by their response were recorded in cassette tapes. The minimum time between stimulus displays was changed to four seconds. Four combinations of two pairs of the stimulus subsets were made to counterbalance the effect of the stimulus sets. The rest of the procedure employed in Experiment 2 was the same as that in Experiment 1. The mean time to complete this experiment was 26 minutes.

\section{Results and Discussion}

Table 2 shows the mean values of observed $d^{\prime}, \beta$, and $p$ (Man). In the second session, the optimal $\beta$ was 3.0 , and the optimal $p(\mathrm{Man})$ and the prior probability of male-height stimuli were .11 and .25 , respectively. The conservative cutoff placement and the probability matching strategy appeared again. These tendencies indicate that the verbalization 
required in this experiment did not alter subjects' performance. Protocol data of one female subject in the second session were analyzed in detail (see Appendix of the present article). Her performance in the first and second sessions was 1.20 and 1.08 , respectively, in terms of $d^{\prime}, 1.14$ and 1.88, respectively in terms of $\beta$, and .46 and .21 , respectively, in terms of $p$ (Man). These values indicate that the subject was one of the typical examples of the numerical decision task, and she verbalized neither more nor less than any other subject.

In No. 4 and No. 11 of her protocol, the subject said that when a presented five-digit number was ambiguous, she would judge it to be a woman's height. It seems difficult for the subject to set an appropriate concrete numerical criterion corresponding to asymmetrical prior probabilities. She used the ambiguous impression of the presented stimulus for determining her response. In the second session, more points could be gained if the subject reduced the number of maleheight responses, because the optimal $p$ (Man) was less than the observed $p$ (Man). The subject attempted, however, to match the rate of her male-height responses to the prior probability of male-height stimuli (No. 40 and 48 in her protocol), a similar strategy to one which another subject had taken which was to count down throughout the session the number of the remaining male-height stimuli. She made maleheight responses considering how recent the last male-height appeared (No. 17, $28,35,40,53,56,68$, and 84 in her protocol). She seemed to be satisfied to increase the accuracy of her responses for both male- and female-height stimuli.

Although the other subjects' protocols were not exactly the same as hers, they did not seem to contain any crucial difference.

\section{General Discussion}

The conservative cutoff placement to the unbalanced prior probabilities was observed in the numerical decision task where subjects were required to make a binary decision to a presented number. The average values of observed $\beta$ did not sufficiently attain the values of the optimal one, as the prior probabilities of stimulus distributions were asymmetrical. This conservatism on the cutoff placement was greater in the low discriminative condition than in the high discriminative condition when measured in $\beta$. A different measure of cutoff locations that was called the critical point also indicated that when prior probabilities were uneven, shifts of cutoff locations were insufficient in the low discriminative condition. Assuming that the subjects used a static criterion throughout a session, responses which contradicted this criterion appeared in all of the sessions. The reaction times for correct responses were longer at the boundary of male- and female-height categories. The probability matching rule was also observed. Observed response probabilities were almost equal to the prior probabilities in both conditions, but did not attain the optimal response probabilities.

Protocol data confirmed the conservative cutoff placement and the probability matching strategy. When prior probabilities were uneven, one subject used her ambiguous impression of a stimulus as a basis for her response bias. At the same time, the subject had an inclination to respond to both stimulus distributions as accurately as possible.

Healy and Kubovy (1977) reported that in their second experiment, the subjects immediately shifted the values of $\beta$ in the low discriminative numerical decision condition rather than in the high discriminative numerical decision condition. These results contradicted the results of Experiment 1 in the present study. The mean value of observed $\beta$ for the first relative block position in the high discriminative condition of their 
experiment with equal prior probabilities is too high (1.94). The means of observed 3 for the other relative block positions, however, show the pattern consistent with the results of Experiment 1 in the present study.

The smaller the degree of discriminability between stimulus distributions, the smaller the shift in $\beta$ (see Equation 3 ). In addition, under low discriminative conditions a large part of the stimulus distribution which has low prior probability is contained in the other stimulus distribution which has high prior probability. Since the subjects have a tendency to respond as accurately as possible to both stimulus distributions, it becomes difficult to shift their criterion for uneven prior probabilities in low discriminative conditions.

Kubovy (1977) found that high posterior probabilities were overestimated and low posterior probabilities were underestimated, and proposed that this distortion of estimated posterior probabilities was one possible basis for the conservative cutoff placement. However, it seems that the estimation of posterior probabilities reflected the subjects' confidence in their responses to the stimuli. Data obtained in the experiments of the present study showed that the conservatism could be due to the heuristics the subjects used for their judgments.

The subjects did not use a unique and static numerical criterion to make their response. Each trial in the task would contain several internal decisions. Zakay (1985) reported the negative correlation between the level of post-decisional confidence and the amount of conflicts experienced in a choice process. When presented five-digit numbers are large or small enough to evoke only one response category without any conflict, subjects may have confidence in their responses. This confidence allows the subjects to proceed to the next state without considering any other probabilities. When the presented numbers belong to the borderline regions of stimulus distributions, these numbers evoke conflicting responses, and other related information such as prior probabilities or the preceding occurrences of malc-height stimuli becomes salient. In such situations, it seems that the subjects have a tendency to take time to seek the solution, and that this solution is based either on the implicit comprehension of the task that responses to both stimulus categories should be accurate or on the strategy for responding to ambiguous stimuli.

Fodor (1983) argued that there were two properties of confirmation in a central process such as decision making. The first is that the facts relevant to the confirmation of a hypothesis may be drawn from anywhere, and the second is that the degree of confirmation assigned to any given hypothesis is sensitive to properties of the entire belief system. These two properties correspond to the findings in the present study. When ambiguous stimuli were presented, dependency on other information relevant to the task became salient and the response which was consistent with subjects' understanding of the task (a part of their belief system) was searched. In decision making, these two properties emerge when the subjects are aware of the ambiguity; this awareness induces them to confirm their response to resolve the conflict.

\section{References}

Ericsson, K.A., \& Simon, H. A. 1980 Verbal reports as data. Psychological Review, 87, 215251.

Fodor, J. A. 1983 The modularity of mind. Cambridge, Massachusetts, and London: MIT Press.

Green, D. M., \& Swets, J.A. 1966 Signal detection theory and psychophysics. New York: John Wiley and Sons, Inc.

Healy, A. F., \& Kubovy, M. 1977 A comparison of recognition memory to numerical decision: How prior probabilities affect cutoff location. Memory \& Cognition, 5, 3-9. 
Healy, A. F, \& Kubovy, M. 1978 The effect of payoffs and prior probabilities on indices of performance and curoff location in recognition memory. Memory 8 Cognition, 6, 544-553.

Healy, A.F., \& Kubovy, M. 1981 Probability matching and the formation of conservative decision rules in a numerical analog of signal detection. Journal of Experimental Psychology: Human Learning and Memory, 7, 344-354.

Hoshino, Y. 1982 Placement of a criterion $\beta$ in a numerical decision task. Proceedings of the $12 \mathrm{th}$ Symposium on Sensory Inspection, 59-64. (In Japanesc)

Kubovy, M. 1977 A possible basis for conservatism in signal detection and probabilistic categorization tasks. Perception \&3 Psychophysics, 22, 277-281.

Kubovy, M., \& Healy, A. 1977a The decision rule in probabilistic categorization: What it is and how it is learned. Journal of Experimental Psychology: General, 106, 427-446.

Kubovy, M., \& Healy, A. $1977 \mathrm{~b}$ Numerical decision and the ideal learner: A reply to Dorfman. Journal of Experimental Psychology: General, 106, $450-452$.

Kubovy, M., Rapoport, A., \& Tversky, A. 1971 Deterministic vs probabilistic strategies in detec-

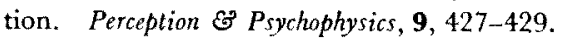

Parks, T. E. 1966 Signal-detectability theory of recognition memory performance. Psychological Review, 73, 44-58.

Sandusky, A. 1971 Signal recognition models compared for random and Markov presentation sequences. Perception \& Psychophysics, 10, 339347.

Sekujer, R., Rubin, E., \& Armstrong, R. 1971 Processing numerical information: A choice time analysis. Journal of Experimental Psychology, 90, 75-80.

Treisman, M., \& Williams, T. C. 1984 A theory of criterion setting with an application to sequential dependencies. Psychological Review, 91, 68111.

Ulehla, Z.J. 1966 Optimality of perceptual decision criteria. Journal of Experimental Psychology, 71, 564-569.

Ward, L. M. 1973 Use of Markov-encoded sequential information in numerical signal detection. Perception \& Psychophysics, 14, 337-342.

Winterfeldt, D. von, \& Edwards, W. 1982 Costs and payoffs in perceptual research. Psychological Bulletin, 91, 609-622.

Zakay, D. 1985 Post-decisional confidence and conflict experienced in a choice process, Acta
Psychologica, 58, 75-80.

(Reccived Nov. 8, 1985; accepted Jan. 18, 1986)

\section{Appendix}

\section{Protocal of the Subject}

No. 1 (stimulus: male, 59416) The same as before, let's say the number is a man.

No. 2 (female, 47458) Ah, because the number is small, woman.

No. 3 (male, 58267) Well, man.

No. 4 (female, 56390) Since there are more women, for the time being, when I'm not sure, I'll judge the number to be a woman.

No. 5 (female, 50220) This is a woman.

No. 6 (female, 54306) Well, woman.

No. 7 (female, 52809) Umm, woman.

No. 8 (female, 53886) Woman.

No. 9 (female, 52157) Woman.

No. 10 (female, 51990) Woman.

No. 11 (female 55811) And, when the number is medium, since I'm not sure, this time, woman's.

No. 12 (female, 53814) This is also a woman. Push.

No. 13 (female, 51940) Woman, push.

No. 14 (male, 55799) Since so far they were mostly women.

No. 15 (female, 46698) Because the number is very small.

No. 16 (female, 50369) Because the number is very small, woman.

No. 17 (female, 56807) Since relatively many women have appeared, for the time being, man, ha.

No. 18 (male, 57802) Ah, man.

No. 19 (female, 52030) Umm, woman.

No. 20 (male, 52779) ...

No. 21 (female, 52632) Umm, but let's take the number for a woman.

No. 22 (female, 46239) Ah, this is absolutely.

No. 23 (female, 53195) Ah, this is also, well, a woman.

No. 24 (female, 53069) Umm, woman, push.

No. 25 (male, 61186) Ah, because the number is too big, man.

No. 26 (female, 58696) Big, ah.

No. 27 (female, 49673) Ah, woman.

No. 28 (female, 56307) Man, because there were very few men, for the time being, ah.

No. 29 (female, 55477) And, for the time being, well, woman. Push. 
No. 30 (male, 60064) Ah, and, because the number is big, man.

No. 31 (male, 53345) For the time being, because the number is small, woman, ah.

No. 32 (female, 51018) Ah, because the number is small, woman.

No. 33 (Female, 45845) Umm, and small, woman.

No. $3 t$ (female, 53642) Umm, woman.

No. 35 (cemale, 56899) Because a man has not appeared, for the time being, man.

No. 36 (male, 54773) Well, small, woman.

No. 37 (fermale, 55358) Well, and well, for the time being, temporary, let's say the number is a woman.

No. 38 (female, 51602) Because this is also rather small, woman.

No. 39 (female, 48962) Ah, because this is also small, woman.

No. 40 (male, 36434 ) Since the ratio is three to one, and there are few men, I think they might appear. Good.

No. 41 (female, 54958) Around this region, woman, umm.

No. 42 (female, 52380) Woman.

No. 43 (female, 52641) Umm, woman.

No. 44 (male, 54976) Umm, for the time being, woman, ah.

No. 45 (female, 51591) Woman.

No. 46 (female, 52208) Woman.

No. 47 (female, 54560) Woman.

No. 48 (female, 56560) Umm, three to one, and for the time being, man, ah, l've missed it.

No. 49 (female, 47284) Because this is small, woman.

No. 50 (female, 50450) Because this is also, well, small, woman.

No. 51 (fermale, 53250) Well, woman.

No. 52 (female, 49088) Because this is smaller, woman.

No. 53 (female, 50652) No man has appeared. Woman.

No. 54 (female, 53056) Umm, this also, for the time being, woman.

No. 55 (female, 50874) Umm, small, woman.

No. 56 (male, 54960) Since there were few men, for the time being, let's take the number for a man.

No. 57 (female, 49915) Woman.

No. 58 (female, 54552) Although a man has not appeared for a while, no, for the time being, woman,

No. 59 (male, 48767) Because the number is small, ah, it was a man.

No. 60 (male, 58879) Because this is, umm, bigger, ah.

No. 61 (male, 55391) Umm, umm, man, since there are more women, ah, I've missed it.

No. 62 (female, 49936) Ah, because this is small, woman.

No. 63 (male, 52394) This is also, well, a woman.

No, 64 (female, 46345) This is, well, a woman.

No. 65 (female, 51581) This is also a woman.

No. 66 (male, 55542) Since there are more woman, for the lime being, woman.

No. 67 (female, 53347) This is, well, a woman.

No. 68 (female, 55684) I wonder if a man has appeared. For the time being, well, man, ha, I've missed it.

No. 69 (female, 52502) For the time being, well, woman. Push.

No. 70 (male, 53494) Umm, woman.

No. 71 (female, 52362) Umm, for the time being, woman.

No. 72 (female, 52401) Ah, woman.

No. 73 (female, 50311) Umm, small, and.

No. 74 (female, 51163) Because this is also small, a girl.

No. 75 (female, 55057) Umm, since there are more women, good.

No. 76 (female, 59959) This is, well, big. Man, oh! I've missed it.

No. 77 (male, 53869) For the time being, woman, oh!

No. 78 (male, 56293) Ah, well, man, since there were few.

No. 79 (male, 51059) For the time being, woman, oh!

No. 80 (female, 52167) Oh! Woman.

No. 81 (female, 50794) Umm, for the time being, because the number is small.

No. 82 (female, 51827) Well, because the number is small, woman. Good.

No. 83 (female, 55880) Since there are more women.

No. 84 (female, 56835) Man, since it has not appeared. 